\title{
PENSER LA NATION. À LA CONFLUENCE DE L'HISTOIRE ET DE LA PHILOSOPHIE
}

\author{
Aurélien Aramini \\ Université de Franche-Comté \\ Carole Widmaier \\ Université de Franche-Comté
}

Quel sens peut prendre une approche philosophique de la nation? II est traditionnellement entendu - et cette lecture n'est bien sûr pas dénuée de légitimité - que la nation est l'affaire exclusive, voire l'invention spécifique du XIX siècle. L'idée de nation serait donc liée, dans son sens même, à une période déterminée de l'histoire ; dans cette perspective, la nation serait impensable avant le XIXe siècle et l'émergence des États-Nations comme type $d^{\prime}$ unité politique, et elle ne pourrait relever, après le xIx siècle, que $d^{\prime}$ 'un avatar nostalgique, c'est-à-dire au fond d'une pure fiction. Tout se passe comme si l'idée même avait accédé à la conscience au sein d'une époque particulière, et cette représentation même nous fait voir que le siècle se trouve ici interprété à partir de l'un de ses propres paradigmes, à savoir la philosophie totalisante de l'Histoire. Si la nation est une réalité historique, inscrite dans un temps et un lieu particuliers, il semble que l'idée de nation ne puisse être pertinente qu'en tant que concept historique ou en tant que concept de théorie politique. Comment pourrions-nous d'ailleurs même imaginer qu'un concept dont le but est de nous permettre de saisir une réalité phénoménale soit susceptible d'avoir une quelconque existence ou une quelconque validité antérieurement à l'événement de l'apparition du phénomène qu'il prétend décrire? Un tel doute, qui a toutes les apparences de la réaction de bon sens, se fonde à la fois sur une conception du langage commun comme pure expression d'une expérience partagée et sur une conception du langage scientifique comme désignation et articulation rigoureuses et claires d'objets définis par abstraction au sein d'une réalité toujours confuse. La question de savoir ce que peut nous dire ou nous permettre de saisir le concept de nation au-delà ou en deçà de sa portée descriptive apparaît donc comme une question vaine. 
Cependant, abandonner une approche philosophique de la nation comme a priori non pertinente consiste à ignorer d'une part qu'un concept $n^{\prime}$ est jamais issu d'une expérience pure et que l'expérience elle-même se trouve toujours déjà prise dans des systèmes de représentations; d'autre part, qu'un concept a toujours lui-même des racines historiques, qu'il ne peut surgir de rien, et qu'il y a donc une histoire des idées : car si la nouveauté du phénomène a pu être légitimement saisie par la nation comme concept, il a fallu pour cela le former à partir de conceptions antérieures dont il a dû être distingué ; enfin, que si le concept de nation peut désigner adéquatement une expérience politique et sociale partagée inédite qui émerge au xIXe siècle, on sait aussi qu'il peut faire l'objet de formes de reprises ou d'assimilations idéologiques, et être dans les expériences collectives identifié à ce à quoi il est en fait uniquement relié ou corrélé, comme en témoigne la multiplicité des investissements de la nation dans des processus de constitution imaginaire de communs politiques. C'est ainsi que l'idée de nation se trouve actuellement politiquement mobilisée, revendiquée ou exploitée au sein de positions, d'intentions et de discours extrêmement divers, qui vont de la mémoire d'une identité commune perdue qu'il conviendrait de retrouver ou à laquelle il s'agirait de donner un nouveau souffle, à l'appel à des formes nouvelles d'autonomisation du collectif qui trouveraient leur inspiration dans des mots d'ordre constitutifs d'une identité, sur le modèle du « som una nació » des mouvements catalanistes.

Dans ce cadre, nous défendons l'idée d'un travail généalogique du concept, qui ne cèdera pas pour autant au fantasme de l'origine, qui soit fondé sur une conception émergentiste de l'histoire, et qui pourra constituer un instrument de lutte contre les périodisations faciles qui simplifient le récit des événements, en en faisant notamment un récit « national ». L'objectif devient alors de penser la nation en articulant les perspectives philologique, historique et philosophique, d'aller chercher dans le passé les sources langagières, conceptuelles et historiques de l'idée de nation; ceci, non pas pour réduire la spécificité de ce que la « nation » vient désigner, mais pour combattre l'illusion d'une sortie du néant du phénomène comme du concept. L'étude de la « nation » est éminemment problématique parce qu'elle se situe précisément à la confluence de trois enquêtes qui possèdent une autonomie relative bien qu'elles s'entrecroisent et interfèrent constamment l'une avec l'autre. La première enquête est celle du philologue qui s'attache à l'ordre linguistique. La «nation » est d'abord un mot qui a une histoire. Étymologique- 
ment, "natio désignait un groupement humain, mais rarement au sens politique du terme ". ' La mise en relation du natio latin avec les termes grecs ethnos, genos et demos introduit une nouvelle difficulté. Si l'on s'attache au «mot » et à ses ramifications, on risque certes d'étendre le sens de la nation bien au-delà de la nation comme "réalité » historique. Mais il n'est pas évident non plus de s'interdire de penser la « nation » en Grèce antique ou à Rome pour la seule raison que la réalité historique de la nation n'existe pas encore : car le mot ou le champ sémantique de la nation existe bel et bien et renvoie à des aspects de la réalité - politique, culturel, biologique synthétisés à des degrés divers dans l'histoire nationale.

La deuxième enquête est celle des historiens et vise à cerner dans l'histoire politique une réalité sociale proprement nationale. Or l'étude de cette réalité, de ses causes et de son sens suppose une interprétation préalable de ce qui doit être identifié comme étant une nation. De fait, il y a des conceptions historiennes de la "nation » qui peuvent éventuellement s'articuler mais qui ne se recoupent pas. Les approches des historiens sont irréductiblement multiples : pensons à la distinction entre nationalisme ethnique et civique de Hans Kohn, à l'étude des processus d'industrialisation et des réseaux de communications chez Karl Deutsch, à l'idée d'une tâche constante de l'éducation pour Elie Kedourie, aux analyses de Benedict Anderson sur les « communautés imaginaires » ou à celles d'Eric Hobsbawm sur les « utopies compensatoires »... En identifiant dans Dioses útiles. Naciones y nacionalismos une véritable "révolution » dans le traitement académique de la " nation », ${ }^{2}$ José Álvarez Junco considère que les travaux modernes des historiens, dans le sillage des études postcoloniales consacrées aux nations et aux nationalismes, conduisent à une double conclusion. D'une part, il s'agit de « relativiser le phénomène, c'est-à-dire, de réduire sa présence à un certain lieu et à un certain moment de l'histoire de l'humanité : I'Europe, à partir des révolutions libérales $»{ }^{3} D^{\prime}$ autre part, il est question de mettre en avant "I'artificialité et l'instrumentalisation politique des identités nationales ». ${ }^{4}$ Pour éviter les «malentendus », Alvarez Junco propose de distinguer trois manières de "comprendre la nation » : la première, "étatique », " possède un contenu strictement politique qui identifie la nation à l'État $» ;$

1 DELANNOI, Gil (2018), La nation contre le nationalisme ou la résistance des nations, PUF, Paris, p. 24.

2 ÁlVAREZ JUNCO, José (2016), Dioses útiles. Naciones y nationalismos, Galaxia Gutenberg, Barcelona.

3 Ibid, p. 22. Toutes les traductions sont d'Aurélien Aramini.

4 Ibid, p. 23.

5 Ibid, p. 42. 
la deuxième, «primordialiste » (primordialista), présente la nation « comme une communauté humaine dotée d'une unité culturelle essentielle ${ }^{6}{ }^{6}$ la troisième, "la plus acceptable » selon l'auteur, est "volontariste » et définit la nation comme « groupe humain caractérisé par sa volonté de constituer une communauté politique ».7 Afin de dépasser ces lectures unilatérales, I'historien propose un concept synthétique de « nation » :

Conjunto de seres humanos entre los que domina la conciencia de poseer ciertos rasgos culturales communes (es decir, de ser un «pueblo » o grupo étnico), y que se halla asentado desde hace tiempo en un determinado territorio, sobre el que cree poseer derechos y desea establecer una estructura política autónoma. ${ }^{8}$

La synthèse ici proposée des différentes approches historiennes ne repose-telle pas en ultime ressort sur le travail philosophique d'élaboration conceptuelle de la nation et de l'articulation de ses deux sens éminents, politique et ethnico-culturel ? S'il n'y a de réalité nationale qu'identifiée conceptuellement, cela signifie-t-il que l'enquête historienne doit être philosophiquement constituée?

La troisième enquête est celle des philosophes. Force est toutefois de constater que la «nation » est quasiment absente de la réflexion philosophique avant le XIXe siècle et la fameuse conférence prononcée par Renan en 1882. Certes, le terme est évoqué par Spinoza ou par Kant mais il n'est central dans aucun système à l'exception notable de Vico, dont la Science nouvelle (1744) s'intitule " science nouvelle relative à la nature commune des nations ». La « nation » pour Vico n'est en aucun cas la « nation » de l'État-nation. La nazione désigne une communauté humaine fondée par les « pères » des premières familles, s'arrachant à l'errance bestiale en instituant les trois principes de l'humanité que sont la croyance en la providence, les interdits relatifs à la sexualité et les sépultures; cette communauté centrée sur

6 Ibid, p. 43.

7 Ibid, p. 44.

8 «Ensemble d'êtres humains entre lesquels prévaut la conscience de posséder certains traits culturels communs (c'est-à-dire d'être un " peuple » ou un groupe ethnique), et qui se trouve installé depuis longtemps dans un territoire déterminé, sur lequel il pense posséder des droits et désire établir une structure politique autonome », ibid, p. 46. 
le « père » et à laquelle vont s'agréger des « serviteurs » affamés, suit un cours (corso) intelligible, une " histoire idéale » ternaire : l'âge des dieux, l'âge des héros et l'âge des hommes. La philosophie vichienne résonnera tout particulièrement au cours du siècle suivant, mais bien moins pour sa conception de la nation que pour sa vision " prométhéenne » de l'histoire, selon laquelle l'homme crée ses dieux et est l'artisan de sa destinée. À l'âge des États-nations, la conceptualisation de la nation à travers les pensées de Fichte ou encore de Renan a conduit à la distinction de deux sens : la nation ethnique et la nation politique. II importe cependant de ne pas les opposer trop radicalement. Dans La communauté des citoyens, ${ }^{9}$ Dominique Schnapper récuse toutes les dichotomies schématiques: conception organiciste vs conception volontariste, conception historique vs conception politique, conception «allemande » vs conception « française ». Cette lecture binaire a finalement conduit à former une vision simplificatrice et donc ambiguë de la nation, alors que Fichte et Renan eux-mêmes, ainsi que d'autres penseurs classiques de la nation, ont précisément tenté d'articuler volonté politique et ancrage historique. Dominique Schnapper souligne alors qu' « interpréter les textes classiques, ceux de Fichte ou de Renan, à l'intérieur de cette opposition, simplifie ou même trahit leur pensée. Fichte, tenant reconnu de la nation "ethnique", intègre dans ses analyses des éléments de la nation civique. Renan, de son côté, $n^{\prime}$ est pas le simple idéologue de la nation-contrat $» .{ }^{10}$ Ainsi le travail philosophique ne conduit pas à simplifier la «nation » en faisant disparaître ses ambiguïtés, mais au contraire à la complexifier en combattant toute tentation de la réduire à un mot ou à une chose.

La nation : à la fois un mot, une réalité politico-culturelle et un concept. Cette diversité $d^{\prime}$ approches - linguistique, historique et philosophique $-n^{\prime}$ indique pas un défaut d'unité ou de méthode. La condition " métisse » de toute réflexion sur la nation est au contraire ce qui doit nous permettre de saisir ce dont la nation précisément vient se distinguer, en inscrivant son idée même dans une constellation conceptuelle qu'il convient, dans l'optique d'une

9 SCHNAPPER, Dominique (1994) La communauté des citoyens, Sur l'idée moderne de nation, Gallimard coll. Folio Essais, Paris,

10 SCHNAPPER, La communauté des citoyens, pp. 236-237. 
appropriation actuelle de la puissance d'agir collective et d'une étude des formes plurielles que peut prendre la représentation d'une identité commune, de considérer non seulement dans son historicité, mais également dans sa synchronicité. C'est ce à quoi s'attachent les études rassemblées dans ce dossier, dont l'objet est d'esquisser une histoire intellectuelle de la «nation », à la confluence de la philologie, de la philosophie et de l'histoire. Ces approches ne sont pas constitutives d'un système d'interprétation cohérent et unitaire, mais elles consonent si l'on admet que les mots, les choses et les concepts possèdent une certaine "souplesse ». Si de très nombreux travaux ont éclairé l'histoire de la " nation » comme réalité politique rigide, nous proposons une autre perspective, proprement conceptuelle, essentiellement centrée sur les types de mises en sens de la « nation » opérées par des philosophes qui s'inscrivent dans un contexte historique déterminé.

Penser la nation à la confluence de la philosophie et de l'histoire, c'est $d^{\prime}$ abord penser la nation avant la nation : en Grèce antique ("L'Autochtonie et la fécondité de la Terre : I'imaginaire politique de la nation naturelle en Grèce ancienne », Arnaud Macé), au sein de l'empire romain (« Inter bárbaros : una propuesta metodológica para descifrar la identidad romana », Coré Ferrer Alcantud) et à l'aube de la modernité ("Quelle pensée de la communauté italienne dans I'humanisme de la fin du XIVe et du début du $X V^{e}$ siècle ?», Laurent Baggioni). Cette pensée de la nation avant la nation donne des points d'appui à un jugement critique sur les appels les plus contemporains à l'unité et à l'identité « nationales». Elle évite à la fois la facilité des grands récits et l'écueil de la projection imaginaire sur le passé des attentes du présent. La portée générale de ces textes consiste en une pluralisation du concept, quand bien même cette pluralisation lui ferait courir le risque de l'éclatement. Dans cette perspective, les études consacrées au $x \mid x^{e}$ et $x x^{e}$ siècles s'attachent à montrer les tensions internes et les difficultés rencontrées par les discours sur la nation, qu'ils soient républicains (« La nation républicaine au-delà des langues premières » Aurélien Aramini) ou marxistes ("Otto Bauer et la question des nationalités » Florian Gulli). Les expériences de l'âge des extrêmes ont pu conduire à renoncer à penser la nation. Plutôt que d'abandonner ce terrain aux nationalismes, ne devraiton pas plutôt envisager une démarche critique visant à comprendre ce dont la nation est venue se distinguer ou se défaire, conceptuellement et politiquement (" Le concept critique de nation de Hannah Arendt à la lumière de sa polémique secrète avec Carl Schmitt »Christian Ferrié) ? Ce dossier contribuera peut-être à nous donner les moyens de nous référer à une idée de la nation et donc de l'identité nationale consciente de soi nourrie par des approches plurielles, historique, philosophique mais aussi ethnologique et anthropologique. C'est tout particulièrement dans cette 
perspective que l'étude comparative du « fait nationalitaire » développée par l'ethnologue Van Gennep au lendemain de la Grande Guerre (« Des rites de passage à la nation : conceptions ethnographiques de la nation chez Arnold Van Gennep (1873-1957) »Vincent Bourdeau) éclaire les débats contemporains sur l'identité nationale en envisageant sa constitution par des rites sociaux dans le cadre d'une conception non substantialiste de la nation.

En raison de l'originalité des articles proposés qui ont assumé de se situer à la confluence de la philosophie et de l'histoire et d'articuler les plans conceptuel et contextuel, nous espérons que ce dossier pourra aussi permettre d'envisager d'autres modalités d'imagination d'un commun sensé, dont les mises en sens philosophiques de pratiques collectives au sein d'époques dans lesquelles la nation n'était encore ni une idéologie, ni un concept, constituent à nos yeux les traces et donc les témoins. 



\title{
L'AUTOCHTONIE ET LA FÉCONDITÉ DE LA TERRE : L'IMAGINAIRE POLITIQUE DE LA NATION NATURELLE EN GRÈCE ANCIENNE
}

\section{THE AUTOCHTONY AND FERTILITY OF THE EARTH: THE POLITICAL IMAGINATION OF THE NATURAL NATION IN ANCIENT GREECE}

\author{
Arnaud Macé \\ Université de Franche-Comté
}

\section{RÉSUMÉ}

Cet article entreprend d'établir que le mythe grec de l'Autochtonie avait pour fonction de répondre aux apories suscitées par l'usage politique de l'idée

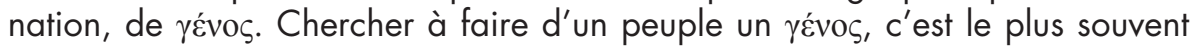
chercher à lui donner un ancêtre commun et lui trouver une affinité originaire avec un territoire. Or ces deux recherches peuvent entrer en conflit : où arrêter sans arbitraire la série des ancêtres ? Comment attester qu'aucun maillon dans la chaîne indéfinie des générations $n^{\prime}$ a jamais migré pour venir s'installer ou ne s'est jamais mêlé à d'autres souches? L'autochtonie, en faisant tout simplement de la terre qu'un peuple habite son plus lointain ancêtre, répond à I'ensemble de ces questions. Nous étudierons la façon dont les discours anciens ont aménagé la conception mythologique d'une déesse Terre procréatrice pour parvenir à cette fin. L'exemple de l'usage platonicien du mythe révélera certaines conséquences inattendues du mythe, comme la réintroduction d'une certaine imprévisibilité dans la nature des hommes et des nations.

Mots-clefs : Autochtonie, Genos, terre, Platon, Hésiode.

\begin{abstract}
This paper attemps to show that the greek myth of Autochtony had the function of solving some of the aporias that met the political use of the idea of nation or $\gamma \varepsilon \dot{v}$ os. Thinking of a people as a $\gamma \varepsilon \dot{v}$ o $\varsigma$ often means to look for a common ancestor and an original connection with a land, two goals that might conflict where to stop the line of ancestors with a good reason? How could one make sure that no generation in the indefinite series of generations ever migrated from somewhere else or mingled with other nations? Autochtony, because it makes of the territory that one people lives in its very own ancestor, settles all issues at once. We will examine how ancient conceptions worked on the myth of the Earth mother to use it towards this end. The example of the platonic use of
\end{abstract}


this myth will also show unexpected consequences, including the reintroduction of some impredictibility within the nature of man and of nations.

Key-words: Autochtony, Genos, Earth, Plato, Hesiod.

\section{RESUM}

\section{L'Autoctonia i la fecunditat de la terra: I'imaginari polític de la nació natural a la Grècia antiga}

Aquest article tracta d'establir que el mite grec de l'Autoctonia tenia la funció de donar resposta a les apories suscitades per l'ús polític de la idea de la

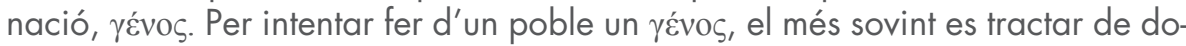
nar-li un avantpassat comú i trobar-lo amb una afinitat original amb un territori. Ara bé, aquestes dues vies poden entrar en conflicte: on ficar fi sense ser arbitraris la sèrie d'avantpassats? Com donar fe de que cap vincle en la cadena indefinida de generacions mai ha emigrat per establir-se o haver-se barrejat amb altres ceps? L'autoctonia, simplement fent que la terra siga una població on ha habitat el seu avantpassat més llunyà, respon a totes aquestes preguntes. Anem a analitzar com els discursos antics han exposat la concepció mitològica d'una deessa procreativa per aconseguir aquest fi. L'exemple de l'ús platònic del mite revelarà certes conseqüències inesperades del mite, com la reintroducció d'una certa imprevisibilitat en la naturalesa dels homes i les nacions.

Paraules clau: Autoctonia, Gènus, terra, Plató, Hesíode. 